\title{
ESTRATÉGIAS DE COACHING E NUDGING E EXCESSO DE PESO: QUAL A RELAÇÃO?
}

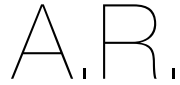
ARTIGO DE REVISÃO

${ }^{1}$ Escola Superior de Tecnologia da Saúde de Coimbra,

Rua 5 de Outubro, 3046-854 Coimbra, Portugal

*Endereço para correspondência:

Daniela Nunes

Escola Superior de

Tecnologia da Saúde

Tecnologia

Coimbra,

5 de Outubro,

3046-854 Coimbra, Portugal

dnunes3218@onutricionistas.pt

Histórico do artigo:

Recebido a 28 de novembro

de 2020

Aceite a 31 de dezembro de 2020

\section{COACHING AND NUDGING STRATEGIES AND OVERWEIGHT: WHAT IS THE RELATIONSHIP?}

Daniela Nunes'; ;oão Lima'; Ana Faria'; Helena Loureiro'

\section{RESUMO}

O excesso de peso continua a ser um problema de saúde pública, sendo, por isso, importante estimular uma mudança do estilo de vida. Todavia há comumente uma forte resistência à mudança que, associada à má gestão de tempo, escolhas alimentares incorretas, falta de disciplina e motivação, comprometem a adesão à terapêutica. A aplicação de estratégias de coaching e nudging parece estar associada ao aumento da motivação, organização e controlo sobre as escolhas alimentares, preparando o doente para uma mudança de comportamento, contribuindo para a diminuição do excesso de peso, melhoria da qualidade de vida, redução da mortalidade e morbilidade e, consequentemente, diminuição dos custos em saúde, embora estudos experimentais sejam necessários para avaliar a eficácia destas estratégias no processo de perda de peso. A aplicação destas estratégias deve ter por base teorias baseadas em evidência científica e aplicadas por pessoal técnico treinado para o efeito, como psicólogos e/ou nutricionistas com formação em coaching aplicado às Ciências da Nutrição, salientando a importância de equipas multidisciplinares para uma eficaz gestão do peso.

\section{PALAVRAS-CHAVE}

Coaching, Excesso de peso, Nudging, Prevenção, Tratamento

ABSTRACT

Excessive weight remains a public health problem and it is therefore important to encourage a change in lifestyle. However, there is usually a strong resistance to change, that combined with poor time management, incorrect food choices, lack of discipline and motivation, compromises adherence to therapy. The application of coaching and nudging strategies seems to be associated with increased motivation, organization and control over food choices, preparing the patient for a change in behavior, contributing to the reduction of excessive weight, improvement in quality of life, reduction of mortality and morbidity and, consequently, decrease in health costs, although experimental studies are needed to evaluate the effectiveness of these strategies in the weight loss process. The application of these strategies should be based on theories, substantiated by scientific evidence and applied by trained technical personnel, such as psychologists and/or nutritionists with training in coaching applied to Nutrition Sciences, emphasizing the importance of multidisciplinary teams for an effective weight management.

KEYWORDS

Coaching, Overweight, Nudging, Prevention, Treatment

\section{INTRODUÇÃo}

A pré-obesidade e obesidade continuam a ser um problema de saúde pública a nível mundial e um fator de risco para o desenvolvimento de outras patologias, nomeadamente diabetes, doenças cardiovasculares, alguns tipos de cancro, doenças respiratórias e distúrbios psicossociais (1-3). Em Portugal, e de acordo com o Inquérito Alimentar Nacional e de Atividade Física 2015-2016, mais de metade da população sofre de pré-obesidade ou obesidade, com prevalências de 34,8\% e 22,3\%, respetivamente (4).

As causas são várias e, por norma, incluem uma interação de diferentes fatores: desequilíbrio entre o consumo alimentar e o gasto energético, sedentarismo e outros fatores ambientais, genéticos, sociais e ambientais $(5,6)$.
É sabido que uma redução da ingestão energética associada ao aumento da prática de exercício físico conduzem à diminuição ponderal, sendo por isso a estratégia mais utilizada para inverter o aumento de peso (3), o que acarreta mudanças nas rotinas do doente. Todavia, verifica-se comumente uma forte resistência à mudança $(7,8)$ que consequentemente irá comprometer a adesão ao tratamento, sendo esta uma das dificuldades fortemente apontada quer por profissionais de saúde quer por doentes $(9,10)$.

A adesão à terapêutica é ainda condicionada por fatores emocionais (os alimentos são frequentemente associados a emoções positivas ou negativas) (6), má gestão de tempo, crenças, falta de disciplina e motivação, frequentemente por 
inexistência de resultados imediatos (9). Embora fosse espectável que o indivíduo fizesse escolhas corretas para resultados positivos a longo prazo, parece que a satisfação imediata prevalece mesmo havendo consciência que isso acarreta efeitos nefastos para a saúde $(11,12)$. Outra dificuldade na perda de peso é a escolha alimentar inadequada, que para além de ser muitas vezes automática e não racional, acresce o facto da oferta de alimentos e bebidas não saudáveis estarem mais disponíveis e a um preço inferior, sendo menos dispendioso optar por alimentos com elevado teor de açúcar e gordura (12-15). É, por isso, importante não só promover uma redução do valor energético ingerido, mas sobretudo estimular uma mudança do estilo de vida (16). Esta alteração, com adoção de hábitos saudáveis, está associada à redução do peso, da massa gorda, da circunferência da cintura e à melhoria do perfil lipídico, glicídico e da pressão arterial (16).

Contudo, para que a mudança de comportamentos ocorra a todos os níveis é fundamental que haja uma intervenção terapêutica multidisciplinar que permita ainda aos doentes identificarem fatores motivadores para a perda ponderal e os capacite para escolher uns alimentos em detrimento de outros, aumentando o autocontrolo na escolha alimentar $(5,13,16)$.

A utilização de técnicas de coaching e estratégias de nudging parece estar associada à adoção e à manutenção de um estilo de vida saudável $(10,11,16-18)$, contribuindo assim para a melhoria da qualidade de vida, redução da mortalidade e morbilidade e, consequentemente, diminuição dos custos em saúde (3, 5, 7, 19-24). Contudo, a aplicação de técnicas e estratégias que envolvem mudanças de comportamento deve ter por base teorias baseadas em evidência científica (5, 8), e aplicadas por pessoal técnico e treinado para o efeito, como psicólogos e/ou nutricionistas com formação em coaching aplicado às Ciências da Nutrição $(1,5,16,25,26)$.

\section{METODOLOGIA}

A pesquisa foi realizada na base de dados eletrónica PubMed entre dezembro de 2019 e agosto de 2020, utilizando os termos "coaching overweight", "nutritional coaching", "nudging food choice" e "nudging overweight". A fim de complementar a pesquisa foram ainda pesquisados os mesmos termos em língua inglesa e portuguesa nas bases de dados SciELO e ScienceDirect. Da pesquisa inicial resultaram 1673 artigos. Foram incluídos artigos publicados entre 2007 e 2020, na língua inglesa, espanhola e portuguesa, realizados em crianças e adultos e excluídos estudos realizados com atletas e/ou enfoque na nutrição desportiva. Os artigos foram selecionados numa primeira fase através da leitura do título, resumo e leitura integral, respetivamente, tendo sido incluídos na análise integral 143 artigos. Desta análise, foram excluídos 109 artigos. Foram ainda incluídos 3 artigos, perfazendo um total de 37, que não seguiram a metodologia supracitada uma vez que foram pesquisados diretamente na fonte, respetivamente Inquérito Alimentar Nacional e de Atividade Física (1 artigo) e Ordem dos Nutricionistas (2 artigos).

\section{Coaching}

De acordo com a International Coach Federation, o coaching é definido como a "relação profissional permanente que ajuda a obter resultados extraordinários na vida das pessoas, na profissão, nas empresas ou nos negócios" (10), sendo o coach e o coachee elementos preponderantes dessa relação, em que o coach é o profissional treinado para desenvolver o processo de coaching, enquanto que o coachee é o indivíduo sujeito a esse processo $(3,9,10,23,25,26)$. Assim, o coaching é um método centrado no coachee, capacitando-o para a resolução de problemas, sem se focar diretamente nesse mesmo problema e sem que o coach Ihe dê respostas, isto é, o coachee com orientação do coach procura as suas próprias soluções $(3,5,9,23,25,26)$.

O objetivo do coaching é, então, conduzir o coachee do seu estado atual (por exemplo, excesso de peso) para um estado desejado (perda ponderal) $(9,20,26)$, funcionando como um facilitador da mudança com o foco em alcançar metas e, capacitando o indivíduo para alcançar o objetivo traçado $(1,3,9,18,23,25)$. A definição dos objetivos e metas é feita pelo coachee sob a orientação do coach, com vista a envolver o coachee no processo de mudança, promovendo o comprometimento, a autorresponsabilização e, consequentemente, a aquisição de disciplina, foco e motivação $(3,9,16,20,25,27)$.

Com base na construção de uma relação de confiança, escuta ativa e técnicas de comunicação, o coach aplica princípios psicológicos e estratégias já estudados, como o modelo transteórico da mudança $(3,19,25)$, para despertar a motivação interior, delinear estratégias e habilidades para que ocorra a mudança, sem que esta seja forçada $(10,16,20)$.

As técnicas de coaching são também utilizadas para que haja uma correta gestão do fracasso $(10,19)$, quando este ocorre, e superação de crenças limitadoras (ideias/pensamentos que impedem o coachee de alcançar o objetivo) (27).

\section{Nudging}

O termo nudge foi definido por Thaler e Sunstein como "qualquer aspeto da arquitetura de escolha que altera o comportamento das pessoas de forma previsível, sem proibir quaisquer opções ou alterar significativamente os seus incentivos económicos" (12). Nudge é então uma alteração no próprio ambiente onde as pessoas tomam as suas decisões com o objetivo de as direcionar para uma escolha, neste caso mais saudável, sem proibir ou eliminar outras opções (menos saudáveis), preservando sempre a liberdade de escolha do indivíduo (11, 28-30). À sua aplicação dá-se o nome de Nudging (13, 29).

Um nudge não conota uma escolha como sendo certa ou errada. $\mathrm{Na}$ verdade estes são praticamente impercetíveis pelo consumidor, que apenas é direcionado para uma alternativa melhor sem imposições ou proibições, sendo exemplos de nudge a disposição de fruta ao nível dos olhos ou a colocação de mais opções saudáveis nas máquinas de venda automática de alimentos em detrimento das opções com elevado teor de gordura e/ou açúcar (13, 30).

Outra característica do nudging é o baixo custo e a facilidade de implementação, o que faz dela uma estratégia interessante e cada vez mais utilizada por profissionais de saúde, nomeadamente nutricionistas e psicólogos, restaurantes, supermercados e governos de alguns países, como o Reino Unido, os Estados Unidos da América e o Brasil (11, 12, $17,24,28,30,31)$.

\section{Aplicação de Estratégias de Coaching e Nudging na Prevenção e Tratamento do Excesso de Peso}

Para que um processo de perda de peso tenha sucesso e mantenha os resultados é necessário adotar um estilo de vida mais saudável, com recurso a novas rotinas (3, 9). É fundamental haver organização do dia a dia e definição de prioridades, sendo o coaching uma estratégia auxiliar para o efeito (3), uma vez que os coaches utilizam os facilitadores para a mudança com vista a ajudar a pessoa que quer prevenir ou tratar o excesso de peso, a superar as ideias limitadoras, dando-lhe ferramentas para controlar o peso a longo prazo, complementando assim o trabalho realizado pelo nutricionista $(1,16)$.

Segundo Stelter, para além da falta de organização, a ausência de autocontrolo é outro obstáculo para a perda de peso (6). O autor refere que coaching na perda de peso trabalha a autorreflexão por parte do doente, promovendo um maior controlo sobre as suas escolhas, 
preparando-o para uma mudança de comportamento, sendo ele o responsável pela mudança e não o profissional $(3,6,8,25)$. Todavia, para que esta mudança de comportamento e alterações de hábitos ocorra é fundamental que sejam delineados objetivos em consulta, que contribuem ainda para que o doente mantenha o foco, motivação e faça a automonitorização $(9,18,25)$. Vários estudos indicam que o coaching aumenta a capacidade de definição de objetivos com vista a melhorar a alimentação, sendo por isso um aliado na consulta de nutrição $(3,8,18$, 25). Para alcançar os objetivos, são delineadas em consulta estratégias para os alcançar, dotando o coachee de ferramentas que o permitem não só emagrecer, mas também manter a perda de peso, libertando-o de crenças limitadoras para a adoção de um estilo de vida saudável e potenciando os comportamentos positivos $(9,25,27)$.

São vários os estudos que têm demostrado que o coaching pode ser benéfico como suporte no processo de perda de peso $(1,8,9)$, contribuindo para uma mudança comportamental com resultados positivos na redução do sobrepeso a curto e longo prazo (5, 9, 32). Esta teoria é ainda suportada pelo Nutrition Care Process, revisto pela American Dietetic Association em 2008, que visa identificar o problema e estabelecer prioridades, metas e estratégias de ação e monitorização na prestação de serviços em nutrição (25).

Sendo o coaching uma ferramenta útil para usar em contexto de consulta de nutrição, o nudging parece ser eficaz fora dela, nomeadamente em cantinas e supermercados, mostrando resultados positivos para a diminuição da prevalência de excesso de peso $(15,30,33)$.

A aplicação de estratégias de nudging parece aumentar, em média, $15,3 \%$ as escolhas alimentares saudáveis que tendem a permanecer no tempo, sendo uma estratégia promissora para a prevenção e redução do excesso de peso a longo prazo (31).

Um estudo de Lopes MB, desenvolvido em cantinas escolares, observou que após a implementação de nudges o consumo de fruta e vegetais aumentou $18 \%$ e $23 \%$, respetivamente e que, tendo em conta o grupo de controle, se verificou um aumento de $83,9 \%$ do consumo de fruta e diminuição de $30,2 \%$ do consumo de sobremesas doces (30). Um outro estudo de Marques et al, publicado em 2020, mostrou que após a intervenção de nudging num bufete, onde o próprio cliente se servia, o consumo de salada aumentou comparativamente com o grupo de controlo (13).

Há diversos exemplos e tipos de nudges estudados na população adulta com idade igual ou superior a 18 anos, em ambos os géneros e em diversos ambientes como cantinas universitárias, refeitórios hospitalares, bufetes, laboratórios e supermercados (11, 13, 14, $24,28,29)$. Os que provam ser eficazes na diminuição da ingesta alimentar ou aumento do consumo de alimentos saudáveis e redução dos alimentos menos saudáveis passam por reduzir o tamanho das porções e embalagens; colocar o valor energético das refeições num menu de restaurante e/ou o tempo necessário, por exemplo de caminhadas, para promover o gasto energético dessa refeição; colocar semáforos nutricionais nos rótulos dos produtos alimentares; expôr frases motivacionais no ambiente onde ocorre a refeição, como bar, refeitório ou cantina; alterar a ordem como os alimentos são apresentados e disponibilizados, colocando os alimentos mais saudáveis num local mais prático e acessível (12, 34).

Vários estudos têm demonstrado que a aplicação de nudges é promissora para a promoção de uma alimentação saudável, sugerindo que estes promovem o aumento de consumo de alimentos saudáveis em detrimento dos menos saudáveis, sendo uma ferramenta útil para a prevenção da pré-obesidade e obesidade e manutenção do peso perdido a longo prazo $(12-15,24,28,29,35)$.

\section{ANÁLISE CRÍTICA E CONCLUSÕES}

As estratégias de coaching e nudging não são um tema recente, embora nos últimos anos tenham sido mais debatidos e, consequentemente, ganho destaque da sua aplicabilidade às Ciências da Nutrição.

A falta de motivação e foco são dificuldades, muitas vezes assinaladas por profissionais e doentes, comprometendo a adesão à terapêutica e, consequentemente, a adesão às consultas de nutrição. Através do coaching é possível delinear estratégias que trabalhem essa falta de motivação e determinação da pessoa com excesso de peso (9). Por sua vez, estratégias de nudging auxiliam na escolha alimentar mais correta, sendo este outro desafio apontado pela pessoa que quer perder peso. Assim, um nutricionista através do coaching com definição de objetivos, construção de uma relação de confiança, escuta ativa, técnicas de comunicação e uma correta gestão do fracasso e do nugding com a redução do tamanho das porções, colocação de semáforos nutricionais nos produtos e exposição de frases motivacionais pode dispor de estratégias que o auxilem no tratamento do excesso de peso, uma vez que há uma associação positiva na interligação destas estratégias. Contudo estudos experimentais são necessários para avaliar a eficácia de ambas no processo de perda de peso.

A aplicação destas estratégias deve não só ser sustentada pela evidência científica, mas também executada por profissionais treinados, como psicólogos e/ou nutricionistas com formação em coaching aplicado às Ciências da Nutrição, ressaltando a importância de equipas multidisciplinares para uma eficaz gestão do peso.

De salientar que é competência de um nutricionista, e citando o Regulamento n. 55/2019, "dominar e aplicar as metodologias de promoção de adesão ao aconselhamento alimentar e à terapêutica nutricional" e "promover a adesão à terapêutica nutricional, ajustando a prescrição sempre que necessário" (36), devendo para isso, e de acordo com o Código Deontológico da Ordem dos Nutricionistas, "utilizar os instrumentos científicos e técnicos adequados ao rigor exigido na prática da profissão, desenvolvendo uma prática informada e conduzida pela evidência científica" (37), para o qual este trabalho pretende contribuir.

\section{REFERÊNCIAS BIBLIOGRÁFICAS}

1. Kleine HD, McCormack LA, Drooger A, Meendering JR. Barriers to and Facilitators of Weight Management in Adults Using a Meal Replacement Program That Includes Health Coaching. J Prim Care Community Health [Internet]. 2019 Jan 31;10:215013271985164. Available from: http://journals.sagepub.com/doi/10.1177/2150132719851643.

2. Jorge R, Santos I, Carraça E, Teixeira VH, Teixeira PJ. Behavioral and psychological predictors of weight loss and long-term weight loss maintenance: a conceptual review of reviews. 2019;17:16-20.

3. Pereira-Lancha LO, Kallas D, Dayan PH, Lancha Junior AH. Técnicas de coaching de bem-estar na mudança do estilo de vida no sistema público de saúde. Estud Avançados [Internet]. 2019 Jan;33(95):235-42. Available from: http://www.scielo.br/ scielo.php?script=sci_arttext\&pid=S0103-40142019000100235\&tlng=pt.

4. Lopes C, Torres D, Oliveira A, Severo M, Alarcão V, Guiomar S, et al. Inquérito Alimentar Nacional e de Atividade Física, IAN-AF 2015-2016: Relatório de resultados [Internet]. Universida. 2015. 291 p. Available from: https://ian-af.up.pt/projeto/objetivos. 5. Karmali S, Ng V, Battram D, Burke S, Morrow D, Pearson ES, et al. Coaching and/ or education intervention for parents with overweight/obesity and their children: study protocol of a single-centre randomized controlled trial. BMC Public Health [Internet]. 2019 Dec 28;19(1):345. Available from: https://bmcpublichealth.biomedcentral.com/ articles/10.1186/s12889-019-6640-5.

6. Stelter R. "I tried so many diets, now I want to do it differently"-A single case study on coaching for weight loss. Int J Qual Stud Health Well-being [Internet]. 2015 Jan 14;10(1):26925. Available from: https://www.tandfonline.com/doi/full/10.3402/ qhw.v10.26925. 
7. Paineau DL, Beaufils F, Boulier A, Cassuto D-A, Chwalow J, Combris P, et al. Family Dietary Coaching to Improve Nutritional Intakes and Body Weight Control. Arch Pediatr Adolesc Med [Internet]. 2008 Jan 1;162(1):34. Available from: http://archpedi. jamanetwork.com/article.aspx?doi=10.1001/archpediatrics.2007.2.

8. Annesi J. From Morbid Obesity to a Healthy Weight Using Cognitive-Behavioral Methods: A Woman's Three-Year Process With One and One-Half Years of Weight Maintenance. Perm J [Internet]. 2012 Oct 30;16(4):54-9. Available from: http://www. thepermanentejournal.org/issues/2012/fall/4907-morbid-obesity-to-a-healthy-weight. html.

9. Mendes FP, Garin NDC, Timm EZ, Ribeiro JL. Aplicação da metodologia coaching para o tratamento da obesidade: uma visão multidisciplinar. Ciência em Mov [Internet] 2018 May 30;19(39):61. Available from: https://www.metodista.br/revistas/revistas-ipa/ index.php/CMBS/article/view/573.

10. Marques-Lopes I. Coaching nutricional, un camino para conseguir cambios. Rev Española Nutr Humana y Dietética [Internet]. 2014 Mar 21;18(1):1. Available from: http://www.renhyd.org/index.php/renhyd/article/view/89.

11. Kwan YH, Cheng TY, Yoon S, Ho LYC, Huang CW, Chew EH, et al. A systematic review of nudge theories and strategies used to influence adult health behaviour and outcome in diabetes management. Diabetes Metab [Internet]. 2020 May; Available from: https://linkinghub.elsevier.com/retrieve/pii/S1262363620300574.

12. Alvarez MJ, Godinho CA. With a little help of a nudge: environmental health regulation. In: Sensos 10. 2015. p. 153-68.

13. Marques ICF, Ting M, Cedillo-Martínez D, Pérez-Cueto FJA. Effect of Impulsivity Traits on Food Choice within a Nudging Intervention. Nutrients [Internet]. 2020 May 14;12(5):1402. Available from: https://www.mdpi.com/2072-6643/12/5/1402.

14. Wilson AL, Buckley E, Buckley JD, Bogomolova S. Nudging healthier food and beverage choices through salience and priming. Evidence from a systematic review. Food Qual Prefer [Internet]. 2016 Jul;51:47-64. Available from: https://linkinghub. elsevier.com/retrieve/pii/S0950329316300210.

15. Tørris C, Mobekk H. Improving Cardiovascular Health through Nudging Healthier Food Choices: A Systematic Review. Nutrients [Internet]. 2019 Oct 18;11(10):2520. Available from: https://www.mdpi.com/2072-6643/11/10/2520.

16. Kent K, Johnson JD, Simeon K, Frates EP. Case Series in Lifestyle Medicine. Am J Lifestyle Med [Internet]. 2016 Nov 8;10(6):388-97. Available from: http://journals. sagepub.com/doi/10.1177/1559827616638288.

17. Thunström L, Nordström J. The Impact of Meal Attributes and Nudging on Healthy Meal Consumption. Mod Econ [Internet]. 2013;04(10):1-8. Available from: http://www. scirp.org/journal/doi.aspx?DOI=10.4236/me.2013.410A001.

18. van Rinsum C, Gerards S, Rutten G, Johannesma M, van de Goor I, Kremers S. The implementation of the coaching on lifestyle (CooL) intervention: lessons learnt. BMC Health Serv Res [Internet]. 2019 Dec 14;19(1):667. Available from: https:// bmchealthservres.biomedcentral.com/articles/10.1186/s12913-019-4457-7.

19. Galvão A. Coaching de saúde e bem-estar na promoção da saúde mental. Rev Port Enferm Saúde Ment [Internet]. 2019 Dec;22(22):5-8. Available from: http://www. scielo.mec.pt/scielo.php?script=sci_arttext\&pid=\$1647-21602019000200001\&lng $=$ pt\&nrm=iso\&tlng=pt.

20. Meya-Molina A, Giménez-Sánchez J. Coaching nutricional como herramienta para la adquisición de hábitos alimentarios preventivos del cáncer. Rev Española Nutr Humana y Dietética [Internet]. 2013 Nov 20;18(1):35. Available from: http://www. renhyd.org/index.php/renhyd/article/view/35.

21. Schwartz RP, Hamre R, Dietz WH, Wasserman RC, Slora EJ, Myers EF, et al. OfficeBased Motivational Interviewing to Prevent Childhood Obesity. Arch Pediatr Adolesc Med [Internet]. 2007 May 1;161(5):495. Available from: http://archpedi.jamanetwork. com/article.aspx?doi=10.1001/archpedi.161.5.495.

22. Young $H-J$, Erickson ML, Johnson KB, Johnson MA, McCully KK. A wellness program for individuals with disabilities: Using a student wellness coach approach. Disabil Health J [Internet]. 2015 Jul;8(3):345-52. Available from: http://dx.doi. org/10.1016/j.dhjo.2014.12.003.

23. Casadei GR, Silva ES, Oliveira LP De, Bennemann RM. Coaching as a strategy for the health promotion of the elderly: a systematic review. Rev Bras Geriatr e Gerontol
[Internet]. 2019;22(4). Available from: http://www.scielo.br/scielo.php?script=sci_ arttext\&pid=S1809-98232019000400302\&tIng=en.

24. Möllenkamp M, Zeppernick M, Schreyögg J. The effectiveness of nudges in improving the self-management of patients with chronic diseases: A systematic literature review. Health Policy (New York) [Internet]. 2019 Dec;123(12):1199-209. Available from: https://doi.org/10.1016/j.healthpol.2019.09.008.

25. Magalhães T, Neves L, Poínhos R. A metodologia de coaching aplicada às Ciências da Nutrição: Usos, potencialidades e controvérsias em Portugal. Acta Port Nutr [Internet]. 2018 Mar 31;12:26-31. Available from: http://actaportuguesadenutricao. pt/edicoes/edicao-no12/.

26. Reis B. Coaching, A arte de gerir Competências. Universidade do Porto.

27. Annesi JJ, Johnson PH, Tennant GA, Porter KJ, Mcewen KL. Weight Loss and the Prevention of Weight Regain: Evaluation of a Treatment Model of Exercise SelfRegulation Generalizing to Controlled Eating. Perm J. 2016;20(3):4-17.

28. Venema TAG, Kroese FM, Verplanken B, de Ridder DTD. The (bitter) sweet taste of nudge effectiveness: The role of habits in a portion size nudge, a proof of concept study. Appetite [Internet]. 2020 Aug;151(March):104699. Available from: https://doi. org/10.1016/j.appet.2020.104699.

29. Bucher T, Collins C, Rollo ME, McCaffrey TA, De Vlieger N, Van der Bend D, et al. Nudging consumers towards healthier choices: a systematic review of positional influences on food choice. Br J Nutr [Internet]. 2016 Jun 28;115(12):2252-63. Available from: https://www.cambridge.org/core/product/identifier/S0007114516001653/type/ journal_article.

30. Lopes MB. Economia comportamental: a aplicação de nudge para a melhoria de hábitos alimentares. Universidade de Brasília; 2018.

31. Velema E, Vyth EL, Hoekstra T, Steenhuis IHM. Nudging and social marketing techniques encourage employees to make healthier food choices: A randomized controlled trial in 30 worksite cafeterias in the Netherlands. Am J Clin Nutr. 2018;107(2):236-46.

32. Haire-Joshu D, Hill-Briggs F. Treating Obesity-Moving From Recommendation to Implementation. JAMA Intern Med [Internet]. 2018 Nov 1;178(11):1447. Available from: http://archinte.jamanetwork.com/article.aspx?doi=10.1001/ jamainternmed.2018.5259.

33. Freeman A. Behavioral Economics and Food Policy: The Limits of Nudging. In: Nudging Health: Health Law and Behavioral Economics. John Hopkins University Press 2016; p. 124-39.

34. Löfgren $\AA$, Nordblom K. A theoretical framework of decision making explaining the mechanisms of nudging. J Econ Behav Organ [Internet]. 2020 Jun; 174:1-12. Available from: https://linkinghub.elsevier.com/retrieve/pii/S0167268120300871.

35. Hanks AS, Just DR, Smith LE, Wansink B. Healthy convenience: nudging students toward healthier choices in the lunchroom. J Public Health (Bangkok) [Internet]. 2012 Aug;34(3):370-6. Available from: https://academic.oup.com/jpubhealth/article-lookup/ doi/10.1093/pubmed/fds003.

36. Ordem dos Nutricionistas. Regulamento N.o 55/2019 da Ordem dos Nutricionistas. Diário da República, 2.a série - N.o 9 - 14 de janeiro 2019 p. 1697-703.

37. Ordem dos Nutricionistas. Código Deontológico da Ordem dos Nutricionistas. Diário da República, 2.a série - N.o 112 - 14 de junho 2016 p. 18664-6. 